\title{
FAST RADIO BURSTS AND RADIO TRANSIENTS FROM BLACK HOLE BATTERIES
}

\author{
Chiara M. F. Mingarelli ${ }^{1,2}$, Janna Levin ${ }^{3,4}$, and T. Joseph W. Lazio ${ }^{5}$ \\ ${ }^{1}$ TAPIR, MC 350-17, California Institute of Technology, Pasadena, CA 91125, USA \\ 2 Max Planck Institute for Radio Astronomy, Auf dem Hügel 69, D-53121 Bonn, Germany \\ ${ }^{3}$ Institute for Strings, Cosmology and Astroparticle Physics (ISCAP), Columbia University, New York, NY 10027, USA \\ ${ }^{4}$ Department of Physics and Astronomy, Barnard College, New York, NY 10027, USA \\ 5 Jet Propulsion Laboratory, California Institute of Technology, Pasadena, CA 91109, USA \\ Received 2015 September 23; accepted 2015 November 9; published 2015 November 23
}

\begin{abstract}
Most black holes (BHs) will absorb a neutron star (NS) companion fully intact without tidal disruption, suggesting the pair will remain dark to telescopes. Even without tidal disruption, electromagnetic (EM) luminosity is generated from the battery phase of the binary when the $\mathrm{BH}$ interacts with the NS magnetic field. Originally, the luminosity was expected to be in high-energy X-rays or gamma-rays, however, we conjecture that some of the battery power is emitted in the radio bandwidth. While the luminosity and timescale are suggestive of fast radio bursts (FRBs; millisecond-scale radio transients) NS-BH coalescence rates are too low to make these a primary FRB source. Instead, we propose that the transients form a FRB sub-population, distinguishable by a double peak with a precursor. The rapid ramp-up in luminosity manifests as a precursor to the burst which is $20 \%-80 \%$ as luminous given $0.5 \mathrm{~ms}$ timing resolution. The main burst arises from the peak luminosity before the merger. The post-merger burst follows from the NS magnetic field migration to the BH, causing a shock. NS-BH pairs are especially desirable for ground-based gravitational wave $(\mathrm{GW})$ observatories since the pair might not otherwise be detected, with EM counterparts greatly augmenting the scientific leverage beyond the GW signal. The EM signal's ability to break degeneracies in the parameters encoded in the GW and probe the NS magnetic field strength is quite valuable, yielding insights into open problems in NS magnetic field decay.
\end{abstract}

Key words: black hole physics - gravitation - gravitational waves - pulsars: general - stars: neutron

\section{INTRODUCTION}

Advanced ground-based interferometers are likely to detect gravitational waves (GWs) from compact binary coalescences within the next few years. Upgrades to aLIGO, e.g., Harry \& the LIGO Scientific Collaboration (2010), are complete and early observation runs have begun. In the coming years, as aLIGO reaches peak sensitivity, it should be joined by VIRGO and KAGRA to create a network of gravitational wave observatories (e.g., Acernese et al. 2008; Somiya 2012). Anticipation of this has motivated a closer look at the landscape of compact binary sources. Encoded in the gravitational waveform is information about the source parameters, ranging from masses and spins to sky location. As such, a diverse and comprehensive toolkit is needed to extract the rich information available from these observations. While some GW source parameters are expected to be very well measured-e.g., the binary chirp mass-the source distance and inclination angle suffer from significant degeneracies, making them difficult to resolve (e.g., Aasi et al. 2013).

One of the most promising tools for lifting the degeneracies in these parameters is the source's electromagnetic (EM) counterpart (e.g., Nissanke et al. 2013). In a neutron star-black hole (NS-BH) coalescence, if the NS is disrupted, then a relativistic jet powered by the rapid accretion of material onto the BH may produce a short gamma-ray burst (GRB; e.g., Metzger \& Berger 2012; Nakar 2007). GRBs are difficult to detect as the beamed emission results in a detection rate of $<1 \mathrm{yr}^{-1}$ with Swift for NS-BH and NS-NS mergers in the aLIGO/VIRGO volume. However, optical and radio afterglows can originate from the jet's interaction with the medium surrounding the burst, lasting days to weeks, or weeks to months, respectively. Another EM counterpart is produced by the radioactive decay of heavy elements synthesized in the ejecta, powering an optical "kilonova," lasting a few days, see Li \& Paczyński (1998).

However, most non-spinning BHs do not disrupt their companion NS before the plunge since the disruption radius, $r_{\text {tidal }}=\left(M_{\mathrm{BH}} / M_{\mathrm{NS}}\right)^{1 / 3} r_{\mathrm{NS}}=13 M_{\odot}$ for $M_{\mathrm{NS}}=1.4 M_{\odot}$ and $r_{\mathrm{NS}}=10 \mathrm{~km}$, is inside the Schwarzschild radius of a BH with $M_{\mathrm{BH}} \gtrsim 7 M_{\odot}$ (slightly larger for a spinning $\mathrm{BH}$ ). Hence, the aforementioned EM follow-up techniques will not be achievable. Moreover, a GW detector with sensitivity comparable to the Einstein Telescope (e.g., Sathyaprakash et al. 2012) is needed to distinguish between NS-BH and $\mathrm{BH}-\mathrm{BH}$ binaries with the same mass ratio if the NS is not tidally disrupted (see Foucart et al. 2013). It is therefore advantageous to have an EM counterpart for a non-disrupted NS-BH binary, enabling the source identification of the GW signal and allowing for independent source parameter measurements (e.g., Tsang et al. 2012).

In this Letter, we describe a transient radio signal, typically lasting a few milliseconds with a luminosity of $\mathcal{L} \sim 10^{40}-10^{41} \mathrm{erg} \mathrm{s}^{-1}$, resulting from a BH interacting with an NS magnetic field in a configuration called a "battery" (see Goldreich \& Lynden-Bell 1969; Lai 2012; McWilliams \& Levin 2011; Lyutikov 2011), which may represent a fraction of the fast radio burst (FRB) population (e.g., Lorimer et al. 2007).

This transient has a distinctive signature: a rapid luminosity increase, manifesting as a precursor given sufficient signal-tonoise ratio $(\mathrm{S} / \mathrm{N})$ and timing resolution, the "burst" from the peak luminosity before merger, and a post-merger burst at least $0.5 \mathrm{~ms}$ after the main burst due to the migration of the NS magnetic field to the $\mathrm{BH}$ and subsequent magnetic field snapping. For a sufficiently bright burst, the NS magnetic field 


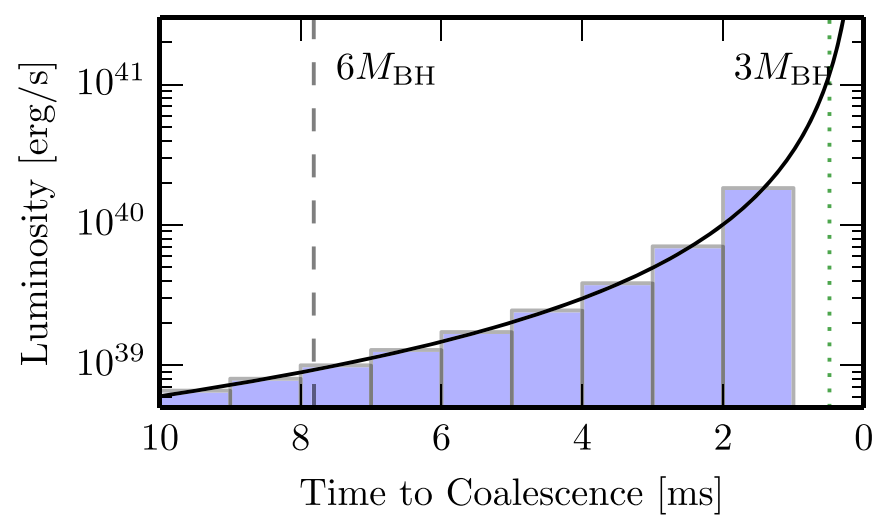

(a) BH Battery luminosity, $1 \mathrm{~ms}$ resolution

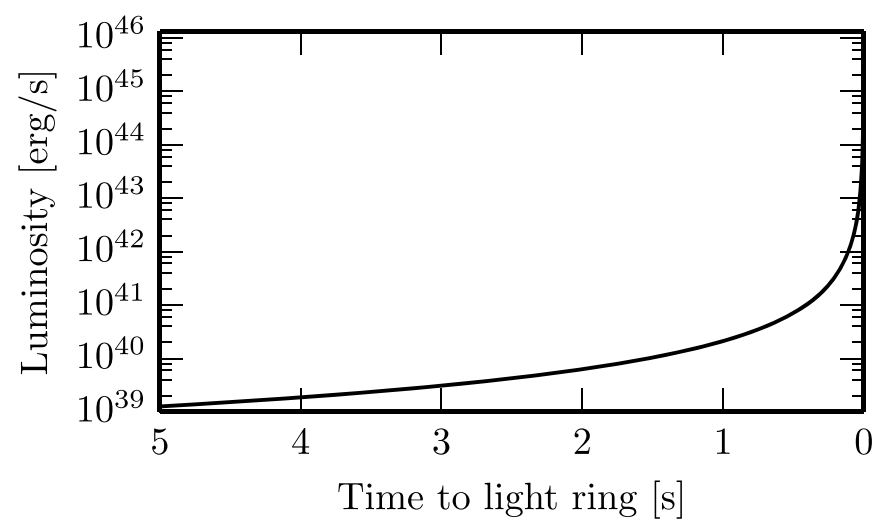

(c) $\mathrm{BH}$ Battery luminosity for $M_{\mathrm{BH}}=10 M_{\odot}, B=10^{15} \mathrm{G}$

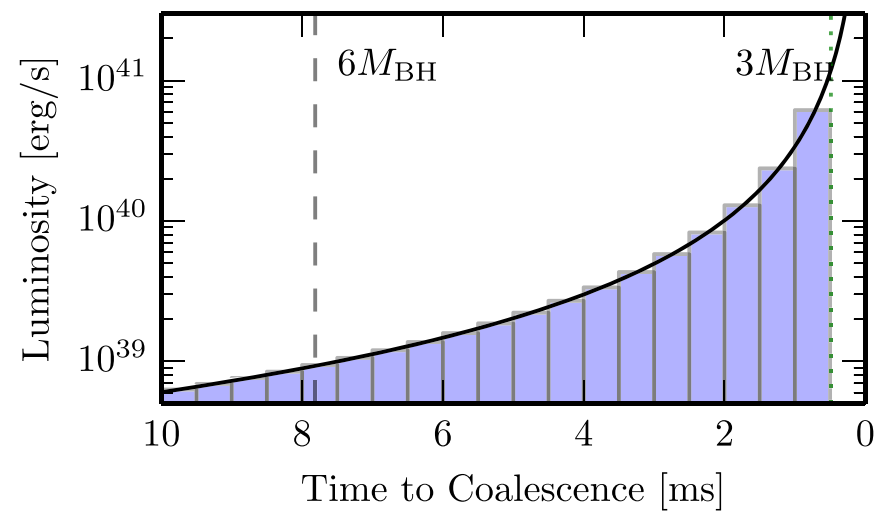

(b) $\mathrm{BH}$ Battery luminosity, $0.5 \mathrm{~ms}$ resolution

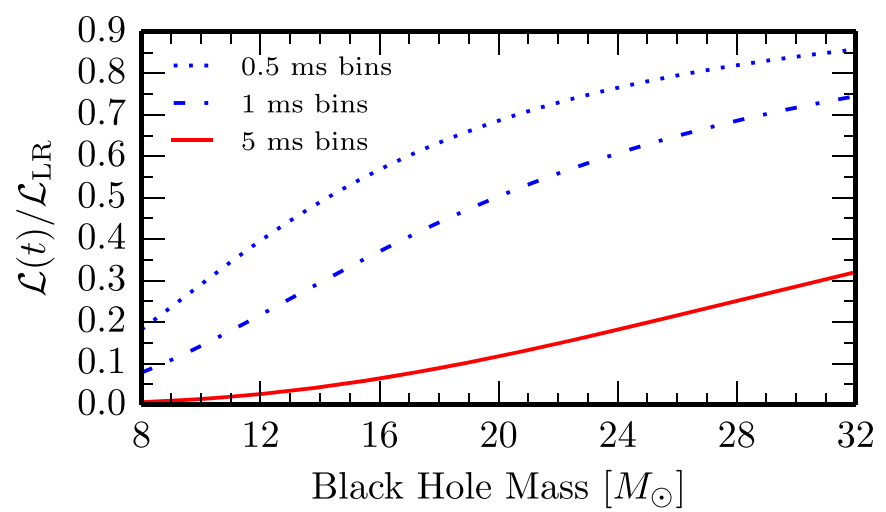

(d) Relative luminosity of precursor to main burst at light ring

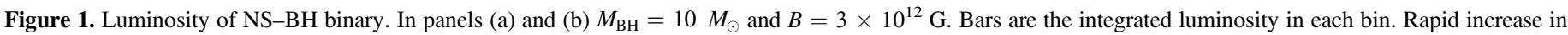

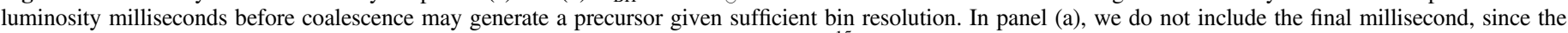

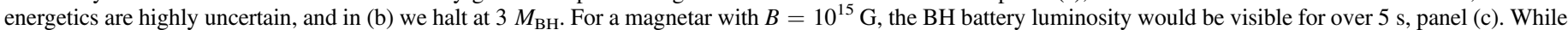

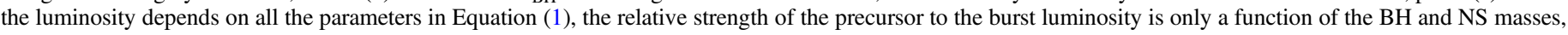

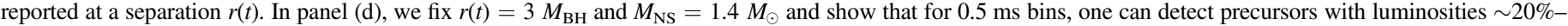

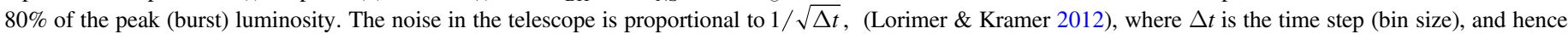
one gains $\sqrt{2}$ in sensitivity moving from 0.5 to $1 \mathrm{~ms}$ bins at the expense of the visibility of the precursor.

strength may be measured from the main and post-merger bursts, granting insights into the long-standing issue of NS magnetic field decay.

\section{THE BH BATTERY}

McWilliams \& Levin (2011) proposed a mechanism to light up a magnetized NS-BH binary for a few milliseconds when the $\mathrm{BH}$ moves through the NS dipole field. In this scenario, the $\mathrm{BH}$ acts like a battery, the NS acts like a resistor with its field lines as wires, and the charged particles in the NS magnetosphere are the current-carriers. The battery is established when the $\mathrm{BH}$ enters the closed magnetic dipole field lines within the light cylinder of the spinning NS, defined as $2 \pi r_{\mathrm{L}} / P=c$ : field lines inside the light cylinder are closed, those passing outside are open (e.g., Goldreich \& Julian 1969). For a spin period of $P=1 \mathrm{~s}$, the battery connects when the pair are separated by $r_{\mathrm{L}} \sim 5 \times 10^{7} \mathrm{~m}$ - thousands of Schwarzschild radii apart for a $10 M_{\odot} \mathrm{BH}$. However, the power may be unobservable until the final stages of coalescence (see Figure 1).

In the late inspiral regime where GWs may be detectable with ground-based interferometers, the magnetic field threading the $\mathrm{BH}$ event horizon will be significant. In this paper, we pursue the conjecture that the mechanism responsible for creating coherent broadband radiation in pulsars can similarly convert a fraction of the energy from the battery into the radio on a short timescale, similar to giant pulses from the Crab pulsar (e.g., Cordes et al. 2004), creating rapid transient radio signals akin to FRBs (e.g., Katz 2015). The radio efficiency parameter $\eta_{\mathrm{r}}$ spans many orders of magnitude and increases with age. Recent radio pulsar observations from Szary et al. (2014) show that the efficiency parameter $10^{-3} \lesssim \eta_{\mathrm{r}} \lesssim 10^{-1}$ is appropriate for pulsars with $\tau \geqslant 10^{7}$ years. Guided by known radio pulsars and in the absence of other theoretical guidance, we adopt a fiducial value of $\eta_{\mathrm{r}}=10^{-2}$.

The luminosity of a $\mathrm{BH}$ battery is a function of the $\mathrm{BH}$ mass, $M_{\mathrm{BH}}$, the magnetic field strength of the NS at the poles, $B_{\mathrm{p}}$, and the NS radius, $r_{\text {NS }}$ (see McWilliams \& Levin 2011):

$$
\begin{aligned}
\mathcal{L}_{\text {battery }}= & 1.2 \times 10^{41} \mathrm{erg} \mathrm{s}^{-1}\left(\frac{\alpha v}{c}\right)^{2}\left(\frac{B_{\mathrm{p}}}{3 \times 10^{12} \mathrm{G}}\right)^{2}\left(\frac{\eta_{\mathrm{r}}}{10^{-2}}\right) \\
& \times\left(\frac{M_{\mathrm{BH}}}{10 M_{\odot}}\right)^{2}\left(\frac{r_{\mathrm{NS}}}{10 \mathrm{~km}}\right)^{6}\left(\frac{r}{30 M_{\odot}}\right)^{-6},
\end{aligned}
$$

where we have fixed the NS mass to be $1.4 M_{\odot}$, $\alpha \equiv \sqrt{1-2 M_{\mathrm{BH}} / r} \quad$ for a non-spinning $\mathrm{BH}$, with $(\alpha v / c)^{2} \sim M / r$. The scaling above also depends on the 
unknown resistivities of the plasma and of the NS. In Equation (1), $r(t)$ is the distance from the surface of the NS, and by substituting $(\alpha v / c)^{2} \sim M / r$, we implicitly assume a point-particle approximation. Since $\mathcal{L}_{\text {battery }} \propto B^{2}$, and $B^{2}$ decays as $r^{-6}$, it is possible that when the BH gets very close to the NS surface that $\mathcal{L}_{\text {battery }}$ could get a boost of orders of magnitude. Hence, Equation (1) is a conservative estimate of the power. A spinning BH will also boost the luminosity, as described in McWilliams \& Levin (2011).

NS magnetic field decay is a long-standing issue in astrophysics. While, for example, Narayan \& Ostriker (1990) and Gonthier et al. (2002) showed that NS magnetic fields can decay on a timescale of $10^{6}-10^{7}$ years, statistical studies, for example, by Stollman (1987), Lorimer et al. (1997), and Bhattacharya et al. (1992) and simulations by Faucher-Giguère \& Kaspi (2006) support decay time constants of $\geqslant 10^{8}$ years. Moreover, recent simulations by Gourgouliatos \& Cumming (2014) show that magnetic field decay in middle-aged NSs is dramatically slowed, and may therefore differ from what is currently drawn on $P-\dot{P}$ diagrams. We therefore take it to be plausible that NS magnetic fields do not significantly decay and we consider $B_{\mathrm{p}} \sim 3 \times 10^{12} \mathrm{G}$ as a fiducial value. In fact, since $\mathcal{L}_{\text {battery }} \propto B_{\mathrm{p}}^{2}$, see Equation (1), EM observations of $\mathrm{BH}$ batteries may provide an avenue to probe the magnetic field strength of old NSs.

\section{GWS FROM NS-BH BINARIES}

Compact binary coalescences are the most promising sources of GWs for ground-based interferometers (e.g., Harry \& the LIGO Scientific Collaboration 2010; Acernese et al. 2008; Somiya 2012). These detectors operate in the high-frequency GW regime with peak sensitivity between 50 and $1000 \mathrm{~Hz}$. Let us consider an NS-BH binary with $M_{\mathrm{BH}}=10 M_{\odot}$ and $M_{\mathrm{NS}}=1.4 M_{\odot}$. Such a binary has a chirp mass of $\mathcal{M}_{\mathrm{c}}=\mu^{3 / 5} M^{2 / 5}=3 M_{\odot}$, where $\mu=m_{1} m_{2} / M$ is the reduced mass of the binary and $M=m_{1}+m_{2}$ is its total mass. We are interested in the last few milliseconds before coalescence, for reasons described in Section 4, and therefore the binary's GW frequency and time to coalescence are reported here at the innermost stable circular orbit of $6 M_{\mathrm{BH}}$. The binary separation $r(t)$ is computed via Peters (1964):

$$
r(t)=\left(\frac{256}{5} \mu M^{2}\right)^{1 / 4}\left(t_{\mathrm{c}}-t\right)^{1 / 4} .
$$

Hence, for a binary $M=11.4 M_{\odot}$ separated by $r(t)=6 M_{\mathrm{BH}}$, the $\mathrm{GW}$ frequency is $470 \mathrm{~Hz}$. The time to coalescence $t_{\mathrm{c}}$ from $6 M_{\mathrm{BH}}$ is obtained via Equation (2):

$$
t_{\mathrm{c}}=7.8 \mathrm{~ms}\left(\frac{r(t)}{6 M_{\mathrm{BH}}}\right)^{4}\left(\frac{\mu}{1.2 M_{\odot}}\right)^{-1}\left(\frac{M}{11.4 M_{\odot}}\right)^{-2}
$$

The detection rates for ground-based interferometers depend on the expected rates of compact binary coalescence events. Due to the lack of direct EM observations of compact binary systems containing BHs, NS-BH rates are based on population-synthesis models (e.g., Abadie et al. 2010). The recent local merger rate for NS-BH binaries with $\mathcal{M}_{\mathrm{c}}=3.2 M_{\odot}$ is 3-20 Gpc ${ }^{-3} \mathrm{yr}^{-1}$ (see Dominik et al. 2015), with an expected detection rate of $1-6 \mathrm{yr}^{-1}$ with aLIGO and $2-15 \mathrm{yr}^{-1}$ using a 3-detector network. For $\mathcal{M}_{\mathrm{c}}=3 M_{\odot}$, the detection probability scales by 0.75 for aLIGO (see Figure 6 of Dominik et al. 2015).

Host identification and parameter estimation are difficult tasks (e.g., Veitch et al. 2015), especially for non-disrupted and non-spinning NS-BH mergers. Numerically, the differences between a $\mathrm{BH}-\mathrm{BH}$ and an $\mathrm{NS}-\mathrm{BH}$ gravitational waveform, orbital evolution, and characteristics of the final remnant cannot be resolved if they have the same mass ratio (see Foucart et al. 2013). In fact, Foucart et al. (2013) claim that only an EM counterpart could prove the presence of an NS in low-spin systems, until the advent of GW detectors with sensitivity comparable to the proposed Einstein Telescope (e.g., Sathyaprakash et al. 2012).

\section{FRBS FROM BH BATTERIES}

FRBs are a recently identified class of single, coherent, millisecond radio pulses (e.g., Lorimer et al. 2007; Keane et al. 2012; Thornton et al. 2013; Ravi et al. 2015; Burke-Spolaor \& Bannister 2014; Spitler et al. 2014; Petroff et al. 2015; Katz 2014). They are marked by the characteristic frequency sweep indicative of propagation through a cold plasma, but with a dispersion measure (DM) or total electron column density suggesting an origin from extragalactic compact objects (e.g., Cordes \& Lazio 2002; Lorimer et al. 2007; Keane et al. 2012; Thornton et al. 2013; Luan \& Goldreich 2014; Ravi et al. 2015). Several candidates have been proposed as FRB sources, including NS mergers (e.g., Li \& Paczyński 1998; Hansen \& Lyutikov 2001; Totani 2013), "supramassive" NS collapse (Falcke \& Rezzolla 2014), and giant pulses or bursts from magnetars (e.g., Popov \& Postnov 2007, 2013).

So far, nine FRBs have been found at Parkes by Lorimer et al. (2007), Thornton et al. (2013), Burke-Spolaor \& Bannister (2014), Ravi et al. (2015), and Petroff et al. (2015), and one was found at the Arecibo Radio Telescope by Spitler et al. (2014). To determine if the hypothesized short-lived burst of radio waves from the battery phase of NS-BH binaries may form a sub-population of FRBs, we examine the energetics of known FRBs in Table 1 and compare to Equation (1).

We take the characteristic flux density of an FRB to be $S_{\nu}=1 \mathrm{Jy}$ and assume that it is at distance of $D=1 \mathrm{Gpc}$ (see Table 1). There is essentially no spectral information currently available on FRBs, with all of the published FRBs having been detected at $1.4 \mathrm{GHz}$. We therefore assume that they are relatively broad band emitters, similar in nature to radio pulsars, with a typical bandwidth $\Delta \nu$ comparable to the frequency of emission (i.e., $\Delta \nu / \nu \sim 1$ ). Finally, with little information available on possible beaming angles, we assume $\Omega \sim 1$ sr. We find that the typical luminosity is then

$$
\begin{aligned}
\mathcal{L}= & 1.3 \times 10^{41} \mathrm{erg} \mathrm{s}^{-1}\left(\frac{S_{\nu}}{1 \mathrm{Jy}}\right) \\
& \times\left(\frac{\Delta \nu}{1.4 \mathrm{GHz}}\right)\left(\frac{\Omega}{1 \mathrm{sr}}\right)\left(\frac{D}{1 \mathrm{Gpc}}\right)^{2} .
\end{aligned}
$$

While FRBs have the required luminosity to have originated from $\mathrm{BH}$ batteries, the rates for FRBs-roughly $2.3 \times 10^{4} \mathrm{yr}^{-1}$ $\mathrm{Gpc}^{-3}$ (e.g., Totani 2013)—are three orders of magnitude larger than the optimistic estimate of an NS-BH merger rate of $20 \mathrm{yr}^{-1} \mathrm{Gpc}^{-3}$ (see Dominik et al. 2015). Our claim is not that all FRBs originate from $\mathrm{BH}$ batteries, rather that there could be multiple populations of FRBs (analogous to the multiple 
Table 1

Luminosities of Reported Fast Radio Bursts (See Equation (4)) Are Comparable to Equation (1)

\begin{tabular}{|c|c|c|c|c|}
\hline & Distance (Gpc) & $S_{\nu}(\mathrm{Jy})$ & $\mathcal{L}_{\text {radio }}\left(\mathrm{erg} \mathrm{s}^{-1}\right)$ & Reference \\
\hline FRB 010724 & 1.0 & 30 & $5.03 \times 10^{43}$ & Lorimer et al. (2007) \\
\hline FRB 110220 & 2.8 & 1.3 & $1.71 \times 10^{43}$ & Thornton et al. (2013) \\
\hline FRB 110703 & 3.2 & 0.5 & $8.58 \times 10^{42}$ & Thornton et al. (2013) \\
\hline FRB 131104 & 1.0 & 2.0 & $3.35 \times 10^{42}$ & Ravi et al. (2015) \\
\hline FRB 110627 & 2.2 & 0.4 & $3.24 \times 10^{42}$ & Thornton et al. (2013) \\
\hline FRB 120127 & 1.7 & 0.5 & $2.42 \times 10^{42}$ & Thornton et al. (2013) \\
\hline FRB 140514 & 1.7 & 0.47 & $2.28 \times 10^{42}$ & Petroff et al. (2015) \\
\hline FRB 011025 & 2.1 & 0.3 & $2.22 \times 10^{42}$ & Burke-Spolaor \& Bannister (2014) \\
\hline FRB 121102 & 1.0 & 0.4 & $6.70 \times 10^{41}$ & Spitler et al. (2014) \\
\hline FRB 010621 & 0.7 & 0.4 & $3.28 \times 10^{41}$ & Keane et al. (2012) \\
\hline
\end{tabular}

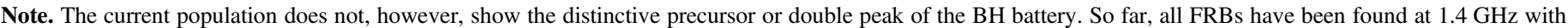

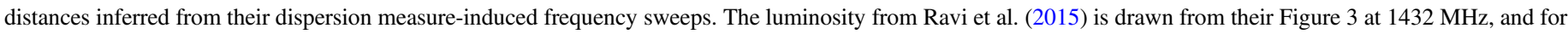
the Keane et al. (2012) burst we fix $h=0.7$ for illustrative purposes.

populations-long and short-for GRBs), with some of the FRBs being radio counterparts to non-disrupted NS-BH binary coalescence, possibly associated with a GW event.

While these sources may be rare, the Canadian Hydrogen Intensity Mapping Experiment (CHIME) is being outfitted with a fast-detection backend with projected rates approaching $10^{4} \mathrm{yr}^{-1}$ (V. Kaspi 2015, private communication), and a proposed augmentation of the Very Large Array's processing capability would enable discovery rates approaching $10^{3} \mathrm{yr}^{-1}$ (C. Law 2015, private communication). A modest estimate is that as many as 5000 FRBs could be discovered over the next 5 years.

The fast radio transients we describe are distinctive. First, there is a ramp-up in the luminosity due to the NS orbiting and plunging into the $\mathrm{BH}$. The continuous luminosity increase may appear as at least one precursor when binned (see Figure 1). We estimate the luminosity until the light ring at $3 M_{\mathrm{BH}}$, since the applicability of Equation (4) breaks down at close separations. In fact, though still winding, the NS begins to plunge between $6 M_{\mathrm{BH}}$ and $3 M_{\mathrm{BH}}$. However, in principle, the power only surges as the NS approaches the horizon. In fact, McWilliams \& Levin (2011) show that systems with significant BH spin are even more luminous than non-spinning ones.

The relative strength of the precursor to the burst is easily computed: $\mathcal{L}(t) / \mathcal{L}_{0}=\left[r(t) / r_{0}\right]^{-7}$, and substituting Equation (2) gives $\mathcal{L}(t) / \mathcal{L}_{0}=\left(\Delta t / \Delta t_{0}\right)^{-7 / 4}$, where $\Delta t=t_{\mathrm{c}}-t$. For example, we compare the luminosity of a canonical $10 M_{\odot}$ $\mathrm{BH}$ and a $1.4 M_{\odot} \mathrm{NS}$ BH battery at the light ring to the luminosity $0.5 \mathrm{~ms}$ earlier. Using Equation (3), $t_{\mathrm{c}} \simeq 0.5 \mathrm{~ms}$ and $\mathcal{L}(t) / \mathcal{L}_{0}=0.29$. The luminosity of the precursor is therefore $\sim 30 \%$ that of the burst. If such a burst were detected with an $\mathrm{S} / \mathrm{N}$ of 30 , the precursor would thus have an $\mathrm{S} / \mathrm{N}=9$. In Figure 1(d), the relative signal strength is so computed for a range of $\mathrm{BH}$ masses. We find that the precursor can be between $20 \%$ and $80 \%$ of the luminosity of the main burst, depending on bin resolution. The current FRB population has been detected with $\mathrm{S} / \mathrm{N} \gtrsim 10$, (e.g., Lorimer et al. 2007; BurkeSpolaor \& Bannister 2014). While none show evidence of a precursor, an even higher $\mathrm{S} / \mathrm{N}$ is likely required to detect the precursor.

The second signature of this FRB sub-population is a postmerger burst from the migration of the NS magnetic field to the $\mathrm{BH}$ and the subsequent violent magnetic field snapping. This second peak is also predicted by the magnetic shock from a supramassive NS collapse into a $\mathrm{BH}$, called a "blitzar" from
Falcke \& Rezzolla (2014). In both the battery and blitzar model, a shock travels outwards and produces radio emission which is in turn modulated by the ringdown of the $\mathrm{BH}$ event horizon, resulting in exponentially decaying sub-ms radio pulses. For the battery model, the delay between the main FRB and the post-merger burst is at least the sum of the time to coalescence and the light-crossing time of the BH, e.g., for $M_{\mathrm{BH}}=10 M_{\odot}$ is $\sim 500 \mu \mathrm{s}+50 \mu \mathrm{s}>0.5 \mathrm{~ms}$. It is probably much longer since the magnetized $\mathrm{BH}$ could retain a magnetosphere which itself supports the magnetic field for longer, e.g., Lyutikov (2011). This admittedly naive estimate reinforces the importance of the $0.5 \mathrm{~ms}$ resolution, so that one can in principle resolve even this (these) post-merger burst(s) from magnetic field snapping and $\mathrm{BH}$ ringdown.

The luminosity of the post-merger peak depends on the NS period $t_{\mathrm{NS}}$, magnetic field strength, and radius, as well as the fraction of magnetic field energy available for the burst, $\eta_{\mathrm{B}}$ (see Equation (4) of Falcke \& Rezzolla 2014), and is potentially as bright as the main burst:

$$
\begin{aligned}
\mathcal{L}_{\text {post }} \simeq 3.8 & \times 10^{41} \mathrm{erg} \mathrm{s}^{-1}\left(\frac{\eta_{\mathrm{B}}}{0.05}\right)\left(\frac{B_{\mathrm{p}}}{3 \times 10^{12} \mathrm{G}}\right)^{2} \\
& \times\left(\frac{r_{\mathrm{NS}}}{10 \mathrm{~km}}\right)^{3}\left(\frac{t_{\mathrm{NS}}}{1 \mathrm{~s}}\right)^{-1} .
\end{aligned}
$$

\section{DISCUSSION}

The population of FRBs described here may have a double peak as well as a precursor: the precursor is from the binned ramp-up in luminosity, followed by the main burst at maximum luminosity, and a post-merger burst due to magnetic field shock. Figure 1 shows examples of the luminosity of the $\mathrm{BH}$ battery milliseconds before coalescence. When observed with sufficient time resolution, $\sim 0.5 \mathrm{~ms}$, and $\mathrm{S} / \mathrm{N}$, the notable precursor feature emerges. The post-merger burst can manifest due to the migration of the NS magnetic field to the $\mathrm{BH}$ at the time of coalescence and the subsequent magnetic field shock, similar to a FRB from the "blitzar" model. This post-merger burst would occur at least $\sim 0.5 \mathrm{~ms}$ after the main FRB, for a $10 M_{\odot} \mathrm{BH}$, and depending on the NS's intrinsic parameters, and may be as luminous as the main burst (see Equation (5)). While we consider a non-spinning $\mathrm{BH}$ in this study, spin would make the system even more luminous and change the innermost stable circular orbit from $6 M_{\mathrm{BH}}$ to $M_{\mathrm{BH}}$, if prograde. 
The luminosity ramp-up itself may contain some structure pulsed at the NS orbital frequency. This would be most interesting immediately before merger, thus at the millisecond level, and would therefore require a very strong EM signal to be detectable.

The distance to an FRB is estimated using the DM, and are in fact upper bounds with an uncertainty of around $20 \%$ (e.g., Thornton et al. 2013). If the sources are closer, then the radio emission efficiency and other parameters in Equations (1) and (4) could be significantly smaller.

The most recent merger rates for NS-BH systems with $\mathcal{M}_{\mathrm{c}}=3.2 M_{\odot}$ range from 3 to $20 \mathrm{Gpc}^{-3} \mathrm{yr}^{-1}$, of which $2-15 \mathrm{yr}^{-1}$ are expected to be detected via GW emission with a 3-detector network (see Dominik et al. 2015). If $\mathcal{M}_{\mathrm{c}}=3 M_{\odot}$, $75 \%$ of these sources are expected to be detected. Some of these may have EM counterparts from the NS disruption, while the majority do not and may belong to the NS-BH population described here. An EM counterpart from an NS-BH coalescence allows for an independent BH and NS mass measurement, Equation (1), as well for a complementary distance measurement to the source, if other parameters are sufficiently well constrained. In the event of an NS-BH GW detection, a coincident EM detection may be possible via new low-latency pipelines sending out triggers, outlined in, e.g., Nissanke et al. (2013) and Chu et al. (2015), or if the telescope is already pointed at the source. This is most probable for telescopes such as CHIME, which shares $\sim 20 \%$ of the two-detector LIGO network sky localization arc from Kasliwal \& Nissanke (2014, Figure 2). While we rely on sky localization arcs from Kasliwal \& Nissanke (2014), who consider NS-NS binaries, a comparable $\mathrm{S} / \mathrm{N}$ for an NS-BH binary would have similar sky localization prospects. In fact, coincident detection prospects with low-frequency radio arrays are especially tantalizing, as dispersion may delay the radio signal, further enabling a possible LIGO/Virgo trigger (Yancey et al. 2015; Trott et al. 2013).

In addition to the coherent radio emission, we also expect a burst of synchrocurvature radiation in X-rays and/or gammarays, which may be more difficult to detect. However, if the power is heavily reprocessed with longer radio emission timescales-perhaps even much after an initial X-ray or GRB - then we are looking for a new kind of radio transient. Indeed, the brief transient EM radiation from the BH battery may be detectable in many wavelengths, and as such may be detected first and more frequently than the GW signal, thereby informing NS-BH merger rates.

Even without a GW counterpart, the radio transients from NS-BH binaries offer a unique avenue to explore the properties of the cosmic population of NS-BH binaries and potentially measure the magnetic field of old NSs. In the age of transient searches, we encourage observers to consider these fascinating source candidates.

We thank the referee, M. Vallisneri, and S. Nissanke for carefully reading the manuscript. We acknowledge valuable discussions with S. McWilliams, M. Kasliwal, D. Tsang, A. Lommen, F. Pannarale, S. Taylor, J. Ellis, J. Bell-Burnell, and P. Goldreich. C.M.F.M. was supported by a Marie Curie International Outgoing Fellowship within the European Union
Seventh Framework Programme. J.L. thanks the Tow Foundation for their support. J.L. was also supported by a Guggenheim Fellowship. Part of this research was carried out at the Jet Propulsion Laboratory, California Institute of Technology, under a contract with the National Aeronautics and Space Administration. An ipython notebook which reproduces our results is available https://github.com/ChiaraMingarelli.

\section{REFERENCES}

Aasi, J., Abadie, J., Abbott, B. P., et al. 2013, PhRvD, 88, 062001 Abadie, J., Abbott, B. P., Abbott, R., et al. 2010, CQGra, 27, 173001 Acernese, F., Alshourbagy, M., Amico, P., et al. 2008, CQGra, 25, 184001 Bhattacharya, D., Wijers, R. A. M. J., Hartman, J. W., \& Verbunt, F. 1992, A\&A, 254, 198

Burke-Spolaor, S., \& Bannister, K. W. 2014, ApJ, 792, 19

Chu, Q., Howell, E. J., Rowlinson, A., et al. 2015, MNRAS, submitted (arXiv:1509.06876)

Cordes, J. M., Bhat, N. D. R., Hankins, T. H., McLaughlin, M. A., \& Kern, J. 2004, ApJ, 612, 375

Cordes, J. M., \& Lazio, T. J. W. 2002, arXiv:astro-ph/0207156

Dominik, M., Berti, E., O’Shaughnessy, R., et al. 2015, ApJ, 806, 263

Falcke, H., \& Rezzolla, L. 2014, A\&A, 562, A137

Faucher-Giguère, C.-A., \& Kaspi, V. M. 2006, ApJ, 643, 332

Foucart, F., Buchman, L., Duez, M. D., et al. 2013, PhRvD, 88, 064017

Goldreich, P., \& Julian, W. H. 1969, ApJ, 157, 869

Goldreich, P., \& Lynden-Bell, D. 1969, ApJ, 156, 59

Gonthier, P. L., Ouellette, M. S., Berrier, J., O’Brien, S., \& Harding, A. K. 2002, ApJ, 565, 482

Gourgouliatos, K. N., \& Cumming, A. 2014, PhRvL, 112, 171101

Hansen, B. M. S., \& Lyutikov, M. 2001, MNRAS, 322, 695

Harry, G. M. \& the LIGO Scientific Collaboration 2010, CQGra, 27, 084006

Kasliwal, M. M., \& Nissanke, S. 2014, ApJL, 789, L5

Katz, J. I. 2014, PhRvD, 89, 103009

Katz, J. I. 2015, arXiv:1505.06220

Keane, E. F., Stappers, B. W., Kramer, M., \& Lyne, A. G. 2012, MNRAS, 425, L71

Lai, D. 2012, ApJL, 757, L3

Li, L.-X., \& Paczyński, B. 1998, ApJL, 507, L59

Lorimer, D. R., Bailes, M., \& Harrison, P. A. 1997, MNRAS, 289, 592

Lorimer, D. R., Bailes, M., McLaughlin, M. A., Narkevic, D. J., \& Crawford, F. 2007, Sci, 318, 777

Lorimer, D. R., \& Kramer, M. 2012, Handbook of Pulsar Astronomy (Cambridge: Cambridge Univ. Press)

Luan, J., \& Goldreich, P. 2014, ApJL, 785, L26

Lyutikov, M. 2011, PhRvD, 83, 124035

McWilliams, S. T., \& Levin, J. 2011, ApJ, 742, 90

Metzger, B. D., \& Berger, E. 2012, ApJ, 746, 48

Nakar, E. 2007, PhR, 442, 166

Narayan, R., \& Ostriker, J. P. 1990, ApJ, 352, 222

Nissanke, S., Kasliwal, M., \& Georgieva, A. 2013, ApJ, 767, 124

Peters, P. C. 1964, PhRv, 136, 1224

Petroff, E., Bailes, M., Barr, E. D., et al. 2015, MNRAS, 447, 246

Popov, S. B., \& Postnov, K. A. 2007, arXiv:0710.2006

Popov, S. B., \& Postnov, K. A. 2013, arXiv:1307.4924

Ravi, V., Shannon, R. M., \& Jameson, A. 2015, ApJL, 799, L5

Sathyaprakash, B., Abernathy, M., Acernese, F., et al. 2012, CQGra, 29, 124013

Somiya, K. 2012, CQGra, 29, 124007

Spitler, L. G., Cordes, J. M., Hessels, J. W. T., et al. 2014, ApJ, 790, 101

Stollman, G. M. 1987, A\&A, 178, 143

Szary, A., Zhang, B., Melikidze, G. I., Gil, J., \& Xu, R.-X. 2014, ApJ, 784, 59

Thornton, D., Stappers, B., Bailes, M., et al. 2013, Sci, 341, 53

Totani, T. 2013, PASJ, 65, L12

Trott, C. M., Tingay, S. J., \& Wayth, R. B. 2013, ApJL, 776, L16

Tsang, D., Read, J. S., Hinderer, T., Piro, A. L., \& Bondarescu, R. 2012, PhRvL, 108, 011102

Veitch, J., Raymond, V., Farr, B., et al. 2015, PhRvD, 91, 042003

Yancey, C. C., Bear, B. E., Akukwe, B., et al. 2015, ApJ, 812, 168 